Original Research

\title{
Decrease of CD38 expression is linked to increase of solitary plasmacytoma pathological grade: a single institution experience from China
}

\author{
Yueying $\mathrm{Li}^{1, \dagger}$, Jianchun Guo ${ }^{1, \dagger}$, Chunxiu Yang ${ }^{1}$, Jian $\mathrm{Xu}^{1}$, Pei Meng ${ }^{1}$, Shu-Yuan Xiao ${ }^{1,2, *}$ \\ ${ }^{1}$ Department of Pathology, Zhongnan Hospital of Wuhan University, 430071 Wuhan, Hubei, China \\ ${ }^{2}$ Department of Pathology, University of Chicago Medicine, Chicago, IL 60637, USA \\ *Correspondence: syxiao@uchicago.edu (Shu-Yuan Xiao) \\ $\dagger$ These authors contributed equally. \\ Academic Editor: Erika Di Zazzo \\ Submitted: 13 October 2021 Revised: 14 December 2021 Accepted: 16 December 2021 Published: 17 January 2022
}

\begin{abstract}
Introduction: Solitary plasmacytoma (SP) is a rare plasma cell disorder characterized by localized neoplastic proliferation of monoclonal plasma cell. Due to its rarity, further understanding of the spectrum of its clinicopathologic features is needed. Methods: A retrospective analysis of cases from a single institution was conducted. Clinical characteristics of the patients were collected; histopathological and semi-quantitative immunohistochemical analyses were performed. Results: Thirteen cases were identified from our pathology archives, including 4 cases of solitary plasmacytoma of bone (SPB) (30.8\%) and 9 extraosseous plasmacytoma (EP) (69.2\%). The mean age of EP is a decade older than SPB. There is no gender disparity. The most common sites involved are the vertebrae and nasopharynx. Histologically, the tumors can be classified into two grades based on degree of differentiation. Immunohistochemically the tumor cells express CD38, CD138, MUM-1, and exhibit light chain restriction. Ki-67 proliferation index is 30\%. In situ hybridization for Epstein-Barr virus-encoded small RNAs (EBER) is negative in six cases tested. Semi-quantitative immunohistochemical analysis showed decreased integrated optical density (IOD) of CD38 in neoplastic cells. IgH gene rearrangement was identified in two cases. Conclusion: SP is a rare plasmacytoid neoplasm that occurs more frequently in older patients. Diagnosis requires a systematic clinical approach combined with the pathological characteristics of plasmacytoid morphology, immunophenotype and light chain restriction. There are more cases of EP than SPB in our series, which is in contrast to that reported in literature. Results from this study suggest that CD38 is a potential immunohistochemical marker associated with prognosis of SP. Further studies with more cases and longer term follow-up may provide more definitive information on risk of progression from SP to multiple myeloma (MM).
\end{abstract}

Keywords: Plasmacytoma; Solitary; Extraosseous; Immunohistochemistry; CD38

\section{Introduction}

Plasma cell dyscrasia encompasses a spectrum of monoclonally proliferative disorders of plasma cells. At one end of the spectrum lies monoclonal gammopathy of undetermined significance (MGUS) that is indolent, whereas at the other, multiple myeloma (MM), which is a malignancy with aggressive biologic behavior [1]. Within this spectrum, solitary plasmacytomas (SP) occur at a single location with no bone marrow involvement. SP accounts for $1-5 \%$ of all plasma cell neoplasms. The patients lack the CRAB features (increased calcium, renal insufficiency, anemia, or multiple bone lesions) and bone marrow involvement [2-4]. However, SP has a propensity to eventually progress to $\mathrm{MM}$ in some patients [5]. SP can be classified into solitary plasmacytoma of bone (SPB) and extraosseous plasmacytoma (EP) depending on the locations. SPB can involve any bone, but more frequently involves bone with hematopoietic elements such as pelvis, skull, ribs, spine, and femur. Approximately $80 \%$ of EP arises in the submucosa in the head and neck region. Less common sites such as the gastrointestinal tract, respiratory tract, bladder, thyroid, kidney, pancreas, uterus and the central nervous system have also been reported [6-8].

Morphologically SP is characterized by tumor cells with round and eccentrically located nuclei, with dense chromatin cluster surrounded by nuclear membrane, similar to mature plasma cells. Plasmablasts with vesicular nuclei and a prominent nucleolus, or bizarre multinucleated cells may also be prominent [9,10]. Other cytologic variants derive from dysregulated synthesis and secretion of immunoglobulin, leading to intracellular accumulation of intact or partially degraded protein. Based on the criteria described by Bartl et al. [9], six histologic types can be delineated according to cytomorphology, namely, Marschalko type, small cell type, cleaved type, polymorphous type, asynchronous type, and blastic type. These six types have subsequently been combined into three prognostic grades: plasmacytoma (low grade), anaplastic plasmacytoma (intermediate grade) and plasmablastic plasmacytoma (high grade).

Accurate diagnosis of SP is critical for proper management. Cell markers are recommended for confirming the 
diagnosis. The tumor cells usually express CD79a, CD138 and CD38, while CD20 is absent [11,12]. The integral membrane protein $\mathrm{CD} 79 \mathrm{a}$ and $\mathrm{CD} 20$ had been identified as B cells markers over 20 years ago [13]. The CD79a protein is present on surface of $B$ cells throughout their life cycle, while CD20 is expressed on pre-, naive, and mature B cells [14], as well as the majority of B-cell lymphomas [15], but not on plasma cells. CD38 is expressed on normal plasma cells as well as on neoplastic plasma cells in MM and SP. Indeed, $>90 \%$ of cells of MM show surface expression of CD38 [16]. CD38 and its homologue CD157 play dual roles as receptors and ectoenzymes, endowed with complex activities related to signaling and cell homeostasis. The canonical plasma cell marker CD138 (syndecan-1) is highly expressed on myeloma and normal plasma cells as well. Among its many functions, CD138 may act as a multifunctional regulator of cell behavior in tumor microenvironment, including cell adhesion [17], endocytosis [18], macropinocytosis [19], and may translocate to the nucleus [20] to regulates switch between myeloma growth and dissemination [21].

Due to the monoclonal nature, the neoplasm produces either kappa $(\kappa)$ or lambda light $(\lambda)$ chain [22,23]. Certain light chain proteins are prone to form amyloidosis of AL (amyloid light chain) type [24]. CD56, the neural cell adhesion molecule (NCAM), is abundantly expressed in cells of neuronal origin, but also other tissues including hematopoietic-derived cells such as dendritic cells, natural killer (NK) and NKT cells [25,26]. It is also expressed in neoplastic plasma cells but not in normal plasma cells [14].

To our knowledge, up till 2020, only 11 papers focusing on SP cases had been published in English language literature, mostly from the West $[2,4,5,7,22,27,28]$. Thus, a systemic review of the clinicopathologic characteristics of the disease from China would have significant value.

In this study, we aimed to examine SP patients from a single medical center in China and assess the clinicopathological characteristics of this disease.

\section{Materials and methods}

\subsection{Patients}

In this retrospective study, cases of SP between January 2015 and April 2021 were retrieved from the pathology archives of our hospital. Clinical history and findings from physical examination, hematological results including complete blood count (CBC), peripheral smear and bone marrow examination were collected from the clinical charts. The diagnoses of SP were made based on the following criteria: (i) histological evidence of monoclonal plasmocytic solitary bone lesion (in case of SPB) or extraosseous mass lesion (in case of EP), (ii) lack of monoclonal plasmocytic infiltration in bone marrow aspirations and biopsies other than the single lesion in SPB, (iii) absence of osteolytic lesions based on skeletal survey of the spine and pelvis, (iv) lack of evidence of end organ damage, such as CRAB
$[29,30]$

\subsection{Tissue microarray construction}

A representative formalin-fixed, paraffin-embedded (FFPE) tissue block from each case was retrieved. A tissue microarray (TMA) was constructed as described previously [31], using MINICORE PLUS (MNC0100005157, Mitogen, UK). The array contains 5 cases of low-grade SP and 3 cases of intermediate-grade SP. As control, 3 cases of inflammatory polyps, each from the uterine cervix, maxillary sinus and eyelid that contain normal plasma cells were included. An index TMA is shown in Fig. 1.

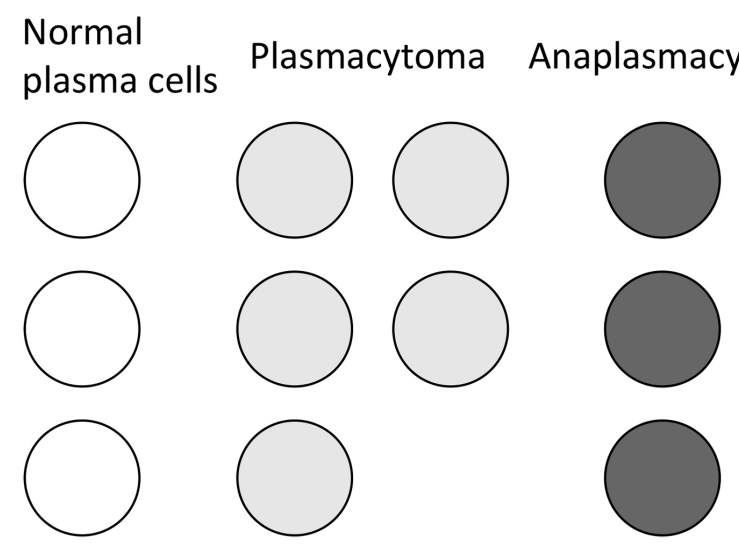

Fig. 1. Schematic map of the index tissue microarray (TMA). There are 8 cases of SP, and 3 cases of mucosal polyps as control for normal plasma cells. SP are grouped according to histologic differentiation into plasmacytoma $(\mathrm{n}=5)$ and anaplasmacytoma $(n=3)$.

\subsection{Immunohistochemistry}

Immunohistochemical stains were performed using a Dako EnVision detection kit, according to the manufacture's instructions (DAKO, Carpinteria, CA). For antigen retrieval, the slides were steamed for 20 minutes in citrate antigen unmasking buffer. The sections were then incubated with the primary antibodies against $\kappa$-light chain, $\lambda$-light chain, MUM1, CD3, CD19, CD20, CD38, CD56 and CD138. These were either rabbit polyclonal antibodies or mouse monoclonal antibodies (DAKO, Carpinteria, CA), at 1:500 or 1:50 dilutions. The incubation was for 45 minutes. Poly-HRP-conjugated anti-mouse/rabbit IgG antibody, as supplied in the EnVison kits, was used as the secondary antibody. The staining was visualized using $3,3^{\prime}$ diaminobenzidine (DAB) (Sigma, Saint Louis, MO, FAST $3,3^{\prime}$ - diaminobenzidine tablets) and slides were counterstained with hematoxylin. In situ hybridization for EpsteinBarr virus-encoded small RNAs (EBER) was performed as described previously [32].

DNA was extracted in formalin-fixed and paraffinembedded (FFPE) sections from SP specimens. Im- 

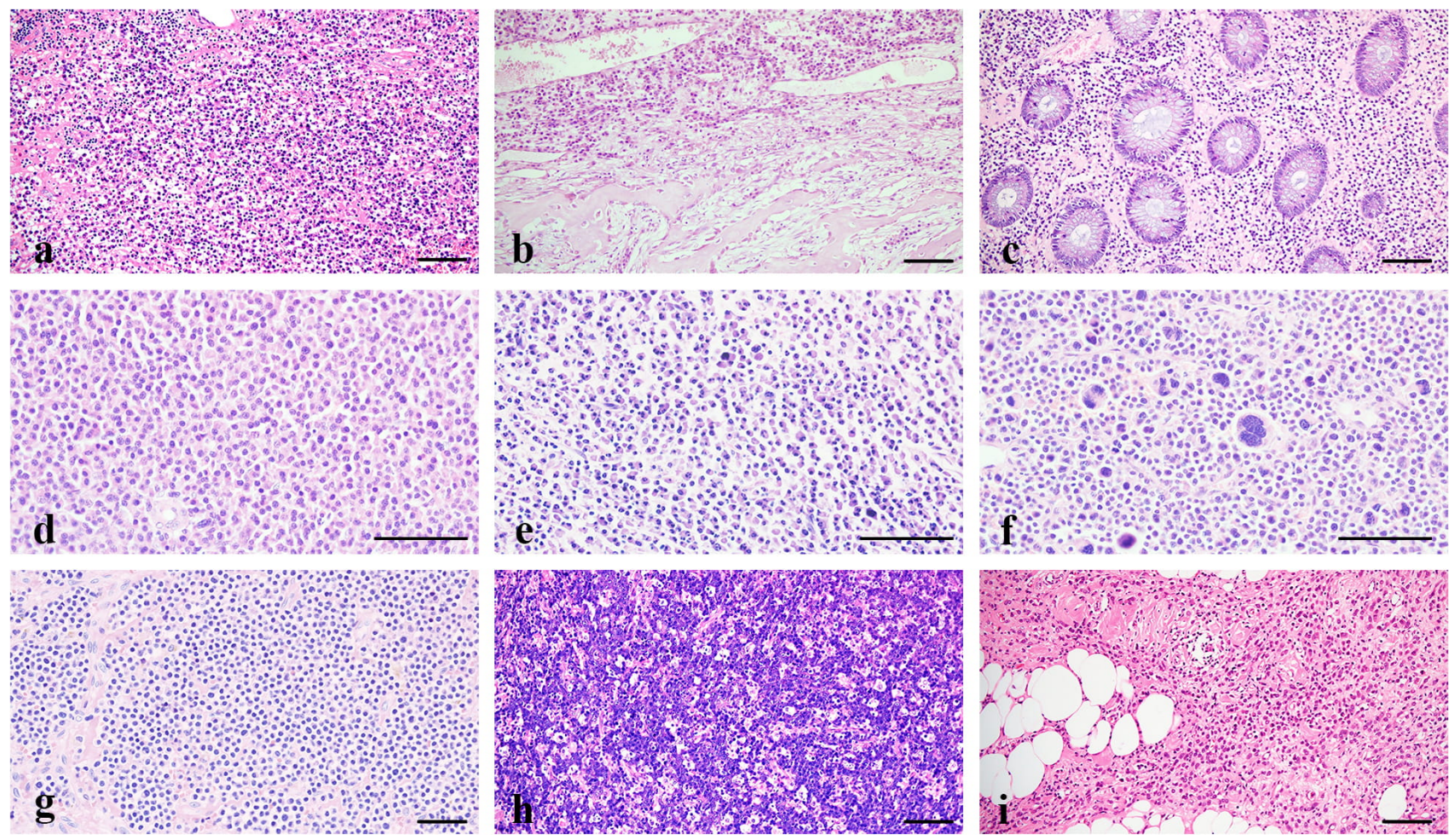

Fig. 2. Comparative histology of SP and controls. $(a, \times 200)$ Normal plasma cells with deep blue cytoplasm, with a pale paranuclear area. The nuclei contain moderate quantities of condensed chromatin, and are eccentrically located. $(\mathrm{b}, \times 200)$ A solitary plasmacytoma in C7 vertebrae: the bone architecture is partially effaced and is infiltrated by neoplastic cells. $(c, \times 200)$ An extraosseous plasmacytoma of colon: neoplastic plasmacytoid cells infiltrating the mucosa and submucosa, with amyloid deposition. (d,e, $\times 400)$ A SPB of lowgrade malignancy: neoplastic cells with eccentric cartwheel nuclei, perinuclear halo and abundant basophilic cytoplasm. (f, $\times 400)$ An anaplasmacytoma with notched, cleaved, or polymorphous nuclei; the ratio of cytoplasmic/nuclear volume is low. $(\mathrm{g}, \times 200)$ A multiple myeloma with morphology indistinguishable from solitary plasmacytoma. (h, $\times 200)$ A plasmablastic lymphoma with a plasmacytoid morphology. (i, $\times 200)$ A breast lobular carcinoma with a plasmacytoid morphology. Scale bar $=100 \mu \mathrm{m}$.

munoglobulin (IG) genes rearrangement analysis was performed with a polymerase chain reaction (PCR)-based technique using BIOMED-2 primer system [33] (Yuanqi Biomedical Technology Inc, China).

\subsection{Quantitative immunohistochemical staining}

The plasmacytoma TMA slides immunohistochemically stained for CD19, CD38, and CD138 were evaluated with the Image. Proplus 6.0 software (Media Cybernetics, Inc., Rockville, MD, USA) and the value of integrated optical density (IOD) was assessed.

\subsection{Statistical analysis}

All data were analyzed using SPSS 19.0 (SPSS Inc., Chicago, IL, USA) and were expressed as mean \pm standard deviation (SD). Comparisons between two groups were performed using Student's $t$-test, while the significance of differences among three or more groups was determined by one-way ANOVA. $p<0.05$ was considered as significant statistical difference.

\section{Results}

\subsection{Clinical characteristics}

As shown in Table 1, 13 cases of SP were identified and included in this study, with seven men and six women. The mean age was 54.2 years (ranging from 23 to 78 years). The cases were classified into 4 SPB and 9 EP. The mean ages of SPB and EP were 46.5 and 57.6 years, respectively. As shown in Table 2, a variety of sites involvement were identified, including the head and neck region (6 cases, $16.7 \%$ cases of SPB and $83.3 \%$ cases of EP), sinonasal or nasopharyngeal area (50\%), gastrointestinal tract (2 cases), central nervous system ( 1 case), and inguinal lymph node ( 1 case). For SPB, long bones were involved in $50 \%$ cases. The most common presentation for SPB is pathological fracture, whereas for EP, soft tissue masses or polyps in the gastrointestinal tract were most common.

\subsection{Pathological characteristics}

As morphological reference, normal plasma cells are 14 to $20 \mu \mathrm{m}$ in diameter. The cytoplasm has a pale paranuclear halo corresponding to the Golgi apparatus and may 
Table 1. Gender and age distributions of solitary plasmacytomas.

\begin{tabular}{|c|c|c|c|c|c|c|}
\hline & \multicolumn{2}{|c|}{ Solitary plasmacytomas } & \multicolumn{2}{|l|}{ EP } & \multicolumn{2}{|c|}{ SPB } \\
\hline & Number of cases & $(\%)$ & Number of cases & $(\%)$ & Number of cases & $(\%)$ \\
\hline \multicolumn{7}{|l|}{ Gender } \\
\hline Male & 7 & 53.8 & 5 & 55.6 & 2 & 50 \\
\hline Female & 6 & 46.2 & 4 & 44.4 & 2 & 50 \\
\hline Total & 13 & & 9 & & 4 & \\
\hline \multicolumn{7}{|l|}{ Age (years) } \\
\hline$<50$ & 5 & 38.5 & 3 & 33.3 & 2 & 50 \\
\hline $50-60$ & 3 & 23.0 & 2 & 22.2 & 1 & 25 \\
\hline$>60$ & 5 & 38.5 & 4 & 44.4 & 1 & 25 \\
\hline Mean \pm SD & $54.2 \pm 16.5$ & & $57.6 \pm 11.5$ & & $46.5 \pm 22.3$ & \\
\hline
\end{tabular}

SD, Standard deviation; EP, extraosseous plasmacytoma; SPB, solitary plasmacytoma of bone.

Table 2. Primary sites of SP.

\begin{tabular}{|c|c|c|c|c|c|c|c|c|}
\hline Case & Age/Gender & Site & Diagnosis & Grade & $\kappa / \lambda$ restriction & EBER & IgH gene rearrangement & Follow-up time \\
\hline 1 & $23 / \mathrm{F}$ & Femur & SPB & Plasmacytoma & $\kappa$ & l & / & $6 y 2 \mathrm{~m}$ \\
\hline 2 & $46 / F$ & Lymph node & EP & Anaplastic plasmacytoma & l & - & l & $6 y$ \\
\hline 3 & $53 / \mathrm{M}$ & Parotid gland & EP & Anaplastic plasmacytoma & $\kappa$ & / & l & $4 \mathrm{y} 11 \mathrm{~m}$ \\
\hline 4 & $40 / \mathrm{F}$ & Rectum & EP & Plasmacytoma & $\lambda$ & - & + & $4 \mathrm{y} 10 \mathrm{~m}$ \\
\hline 5 & $70 / F$ & Nasal cavity & $\mathrm{EP}$ & Anaplastic plasmacytoma & / & - & l & $4 y 6 m$ \\
\hline 6 & $67 / \mathrm{M}$ & Colon & EP & Plasmacytoma & - & / & + & $4 y 3 \mathrm{~m}$ \\
\hline 7 & $57 / \mathrm{M}$ & Humerus & SPB & Plasmacytoma & $\lambda$ & l & l & $4 y 3 m$ \\
\hline 8 & $65 / \mathrm{M}$ & Thigh & $\mathrm{EP}$ & Anaplastic plasmacytoma & $\lambda$ & - & l & $4 y 2 m$ \\
\hline 9 & $55 / \mathrm{M}$ & Parasagittal & EP & Anaplastic plasmacytoma & $\kappa$ & I & l & $4 y 1 \mathrm{~m}$ \\
\hline 10 & $28 / \mathrm{M}$ & Vertebral & SPB & Plasmacytoma & $\kappa$ & I & / & $3 y 8 m$ \\
\hline 11 & $47 / \mathrm{F}$ & Nasal cavity & EP & Plasmacytoma & $\lambda$ & - & l & $2 \mathrm{y} 11 \mathrm{~m}$ \\
\hline 12 & $75 / \mathrm{M}$ & Nasal septum & EP & Plasmacytoma & / & - & l & $1 \mathrm{y} 4 \mathrm{~m}$ \\
\hline 13 & $78 / \mathrm{F}$ & Skull & SPB & Plasmacytoma & $\lambda$ & / & / & $8 \mathrm{~m}$ \\
\hline
\end{tabular}

contain one or more vacuoles. The nuclei contain moderate amount of condensed chromatin, and are eccentrically located (Fig. 2a). Rarely plasma cells can be binucleated or multinucleated. In contrast, as shown in Fig. $2 b$ of a case of rectal plasmacytoma, the neoplastic plasma cells are densely arranged and invade the colonic crypts, with amyloid deposits in the mucosa (Fig. 2c). Based on the criteria described by Bartl et al. [9], 2 histologic categories can be distinguished according to the degree of differentiation, all 4 SPB cases in our series were of low grade malignancies, 4 of the 9 EP were low grade, while 5 were of intermediate grade. The tumor cells in the 8 low grade cases were indistinguishable from normal plasma cells, with eccentric cartwheel nuclei, perinuclear halo and abundant basophilic cytoplasm, and mostly with inconspicuous nucleoli. Some of the low grade tumors were of small-cell variant, characterized by a lymphoplasmacytic appearance with a narrow rim of basophilic cytoplasm and occasional perinuclear halo (Fig. 2d,e). Five EP cases exhibited intermediate grade features, designated as anaplasmacytoma, with most cells having notched, cleaved, or even polymorphous nuclei of various sizes. The ratio of cytoplasmic/nuclear volume is low, but a small perinuclear halo is usually present (Fig. 2f). No cases in our series exhibited high grade features, which include a mixture of relatively normal-appearing plasma cells and plasmablasts-like cells with vesicular nuclei and prominent, centrally located (immunoblasts-like) nucleoli, a moderate rim of basophilic cytoplasm and a faint perinuclear halo [9].

Our cases were distinguished from the following differential diagnosis: (1) MM, although the histological changes of SP overlap with that of MM (Fig. 2g), the latter shows multiple bone destructions, CRAB symptoms, and increasing serum $\beta$-microglobulin; (2) lymphoma with plasma cell differentiation, such as marginal zone, MALToma, plasmablastic lymphoma (Fig. 2h); (3) metastatic carcinoma with a plasma-like morphology, such as lobular carcinoma of breast (Fig. 2i), plasmacytoid urothelial carcinoma. 

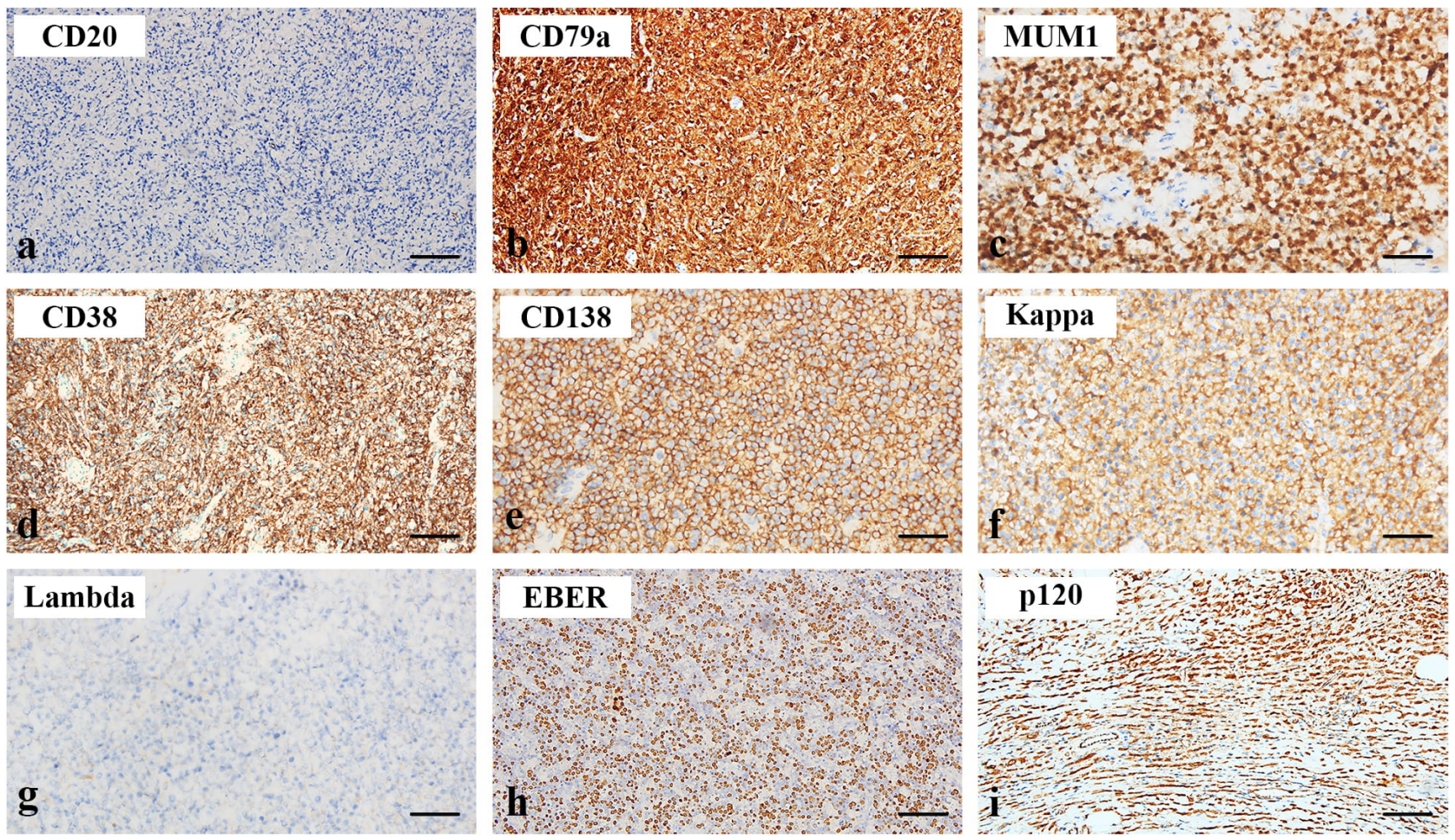

Fig. 3. Immunophenotypes of SP. The neoplastic cells are negative for CD20 (a, $\times 200)$, but with diffuse positivity for CD79a (b, $\times 200)$, MUM-1 (c, $\times 200)$, CD38 (d, $\times 200)$, and CD138 (e, $\times 200)$. F and G: a case of SPB with monoclonal kappa light chain restriction: positive for kappa light chain (f, $\times 200)$, and negativity for lambda light chain $(\mathrm{g}, \times 200)$. $(\mathrm{h}, \times 200)$ in situ hybridization for EBER is positive in a plasmablastic lymphoma. (i, $\times 200)$ A lobular carcinoma of breast expressing P120 in in cytoplasm. Scale bar $=100 \mu \mathrm{m}$.

\subsection{Immunophenotype and gene profiles}

Immunohistochemically, both normal and neoplastic plasma cells are negative for CD20 (Fig. 3a), as expected. Most cells express cytoplasmic CD79a and MUM-1 (Fig. 3b,c), while tumor cells from SP exhibit diffuse membranous CD38 and CD138 expression (Fig. 3d,e). SP cells show light chain restriction, as shown in Fig. 3f,g a case of SPB being kappa positive and lambda negative. The tumors are negative for EBER that differential diagnosis with plasmablastic lymphoma (Fig. 3h), and negative for cytoplasmic P120 in contrast to carcinomas with plasmacytoid features (Fig. 3i). All the cases are negative for Cyclin D1 as well. Eleven cases express MUM-1, and less than 30\% express CD56. Eight cases tested for immunoglobulin lightchain production, 5 of which $(62.5 \%)$ are $\kappa$-chain restricted, while the other $3(37.5 \%) \lambda$-chain restricted. All 6 cases tested for EBER are negative. IgH gene rearrangement is positive in 2 cases (Table 3 ).

\subsection{Validation of the index TMA to evaluate immunohistochemical staining in normal and neoplastic plasma cell}

A TMA was constructed as described in Material and Method in order to examine the immunophenotypic features in normal and neoplastic plasma cells. The array contain 2 groups of specimens (normal plasma cell group, $n=3$; neoplastic plasma cell group, $n=8$ ). It showed positive CD19
Table 3. Immunophenotype feature of SP in our study.

\begin{tabular}{lccc}
\hline IHC maker & Positive & Negative & Not tested \\
\hline CD20 & 0 & 13 & 0 \\
Mum1 & 11 & 1 & 1 \\
CD56 & 1 & 4 & 7 \\
CD38 & 13 & 0 & 0 \\
CD138 & 13 & 0 & 0 \\
EBER & 0 & 6 & 7 \\
\hline
\end{tabular}

in normal plasma cells, but negative in neoplastic plasma cells (Fig. 4a). Both normal and neoplastic plasma cells expressed CD38 and CD138 (Fig. 4b,c). Quantitative analysis showed no significant difference in CD138 expression between the normal $(50.66 \pm 26.85)$ and neoplastic plasma cells (48.06 \pm 23.33 ) based on IOD value, but CD38 was expressed at lower level in neoplastic plasma cell $(67.05$ $\pm 31.36)$ as compare to the normal counterpart (112.88 \pm 3.14) $(p<0.05)$. In addition, CD138 IOD (50.66 \pm 26.85$)$ is significantly lower than CD38 IOD (112.88 \pm 3.14$)$ in normal plasma cells $(p<0.05)$, while there is not significant difference in neoplastic plasma cell between these 2 markers $(67.05 \pm 31.36$ vs $48.06 \pm 23.33)$ (Fig. $4 d)$. The samples were further separated into 3 groups according to their histological grades (normal, $\mathrm{n}=3$; plasmacytoma, $\mathrm{n}$ $=5$; anaplasmacytoma, $\mathrm{n}=3$ ). CD38 was more strongly 
CD19

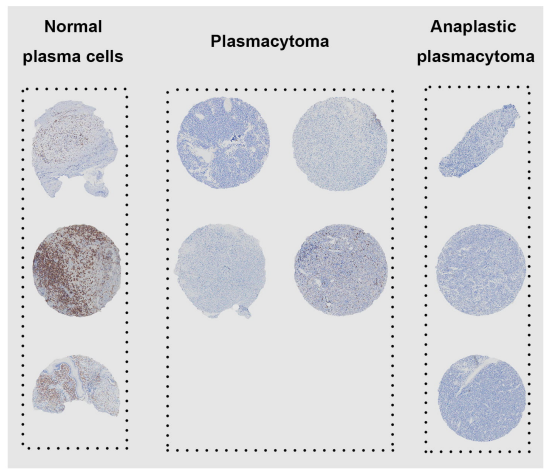

a

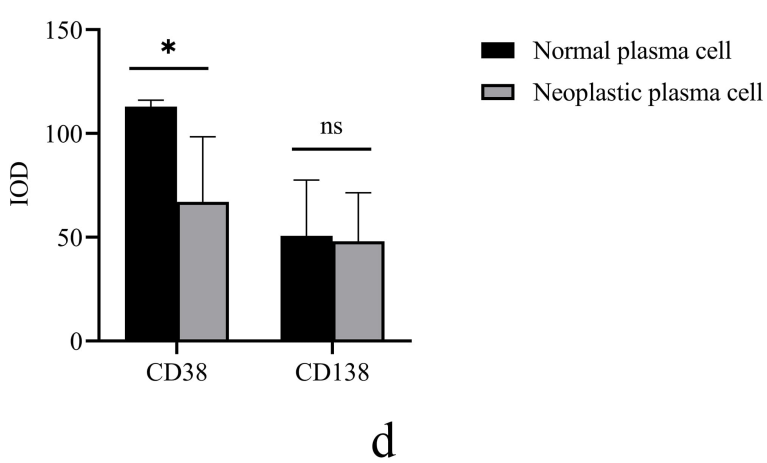

CD38

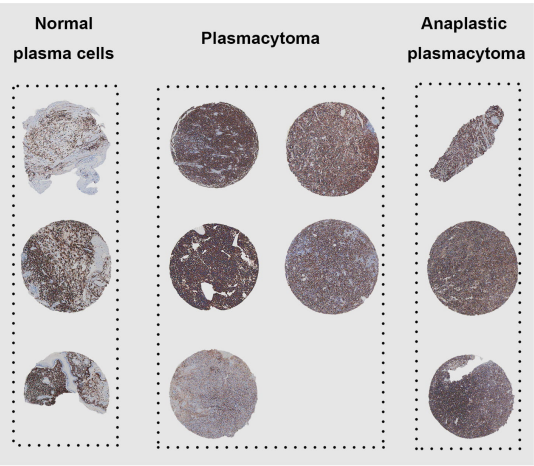

b
CD138

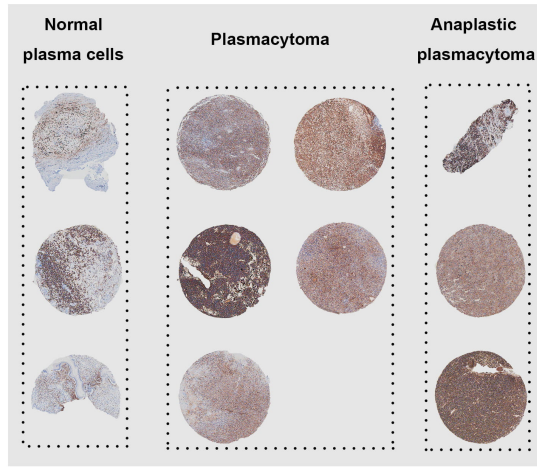

C

Fig. 4. Representative images and quantitative analysis of a standardized TMA. Images of TMA stained immunohistochemically shown at lower magnification ( $\times 40$ ), including (a) CD19, (b) CD38, (c) CD138. (d) Representative histogram shows IOD values of CD38 and CD138 for normal control and SP cases. Normal plasma cells exhibit higher IOD of CD38 than neoplastic plasma cells, while there is no significant difference in IOD of CD138 between normal plasma cells and neoplastic cells. (e) The specimens are separated into 3 groups according histological grades (normal plasma cell, $n=3$; plasmacytoma cell, $n=5$; anaplasmacytoma cell, $n=3$ ). There is a significant decrease of IOD of CD38 with increasing tumor grade. The IOD of CD138 is indistinguishable among the groups. Values are presented as mean $\pm \mathrm{SD} .{ }^{*} p<0.05 ; * * * p<0.001$.

expressed in the intermediate grade plasmacytoma (83.59 $\pm 27.00)$ than in low grade ones $(39.48 \pm 12.60)(p<$ $0.05)$. The IOD of CD138 is not distinguishable among the 3 groups ( $50.66 \pm 26.85$ vs $48.60 \pm 18.72$ vs $47.15 \pm 34.66$ ) (Fig. 4e).

\section{Discussion}

Solitary plasmacytoma (SP) is defined as a localized mass of neoplastic, monoclonal plasma cells. Although SP is morphologically indistinguishable from multiple myeloma (MM), both location and clinical behavior are significantly different between the two entities. Among SP, SPB is reported to be about $40 \%$ more frequent than EP [29]. However, more EP is encountered in our cohort $(69 \%)$, indicating that in the central region of China, distribution of SP is different from that described in the literature from the West. Otherwise, the tumor locations of our cases are similar to those from the literature, with SPB most fre- quently involving the vertebra, and EP, nasopharynx. In addition, we describe an unusual site of EP in lymph nodes, the latter had been rarely reported [8].

Several studies had shown mean age of SP patients at diagnosis ranging from 55 to 60 and a gender disparity for EP $[2,4]$. In our study, no gender disparity is observed. The mean age of EP at diagnosis in our series is 57.6 years which is one decade later than SPB, possibly due to the small number of patients studied. According to the literature, age at diagnosis has a significant role in determining prognosis [29].

Histological evaluation of biopsies is mandatory for diagnosis of SP, with exclusion of other bone lesions and lymphoma [8]. The tumor cells include mature, normal appearing, immature, plasmablastic, and pleomorphic types [9]. About $10 \%$ cases have plasmablastic morphology. Multinucleated, multilobed, pleomorphic plasma cells are prominent in some cases [9]. Although low grade tumors are easily recognized histologically, high grade tumors with 
poorly differentiated cells such as plasmablastic or anaplastic types require special studies. In our series, all cases of SPB are of low grade, while 5 cases of EP are anaplastic plasmacytoma. Testing for monoclonal light chain expression helps for diagnosis. Particularly, kappa or lambda light chain restriction is helpful in differentiating SP from an inflammatory process with enrichment of plasma cells, and lymphomas with prominent plasmocytic differentiation, such as extranodal marginal zone lymphoma (MZL) of mucosa-associated lymphoid tissue (MALT lymphoma). The latter is positive for CD20, while SP is typically CD20 negative. Immunophenotype feature can also distinguish other plasmocytoid tumors, such as some poorly differentiated carcinomas.

SP is usually treated with radiation, surgery or a combination of both. EP is highly radiosensitive. Radiation therapy is currently the first line of treatment for EP with a local control rate reaching 90\%. The 10-year disease-free and overall-survival rates range from $50 \%$ to $80 \%$ [27,28]. EP in the lower respiratory tract is managed by radiation therapy alone, while EP in the upper respiratory tract may be treated with both radiation and surgery. EP in the gastrointestinal tract requires surgical excision [5,34]. Followup time for our cases ranged from 8 to 74 months, with an average of 47.8 months. Up to the end of our study, the patients were either cured or progressed to MM.

CD 38 is a membrane-bound protein of $45 \mathrm{kDa}$ first identified in 1980 [35]. It is a single-chain transmembrane type II glycoprotein encoded by a gene mapped to chromosome 4 [36]. CD38 was historically considered a surface marker for T cells [37]. Recent studies have revealed that it is the main nicotinamide dinucleotide (NAD+) catabolic enzyme, related to NAD+ metabolism [38-41]. As a coenzyme, nicotinamide adenine dinucleotide (NAD+) can directly and indirectly influence many key cellular functions, including metabolic pathways, DNA repair, chromatin remodeling, cellular senescence and immune cell function $[42,43]$. CD38 also modulates extracellular NAD + precursors, and causes a substantial decline in cellular NAD+ levels [41], thus altering the availability of substrates for enzymes regulating cellular homeostasis. In our study CD38 is found to be uniformly expressed at high levels in plasmacytoma, more strongly in low grade plasmacytomas than intermediate grade ones. The prognostic relevance of the histologic categories had been confirmed in a previous study [9]. Our findings suggest that CD38 immunohistochemistry is a clinically useful method of predicting prognosis for SP. Nevertheless, more cases need to be studied to confirm this finding; additionally it may be important to analysis NAD+ level in different tumor grades, to further reveal the role of CD38 as a key modulator for NAD+ metabolism in the context of solitary plasmacytoma.

Existing reports on SP are mainly from the West and India. In this study, we aimed to examine SP patients from China and assess their clinical characteristics, survival, and pathological feature. Most significantly, we have analyzed immunohistochemical characteristics by TMA and shown that CD38 may be a marker related to prognosis of SP. However, duo to the small sample size and short follow-up time, a future study with more samples and longer followup time is necessary to confirm this finding.

\section{Conclusions}

Clinicopathological features of 13 cases of SP are described in this study, with EP more frequently encountered than SPB. Patients with SPB present at an earlier age than EP. Immunophenotypically, CD38 is more strongly expressed in plasmacytoma than in anaplastic plasmacytoma. Although histopathological examination remains the most important method in diagnosing these lesions, a systematic approach is required for definitive diagnoses. Treatment of SP is either radiotherapy, surgery or a combination of both. Since it takes years for plasmacytoma to progress to multiple myeloma, a longer follow-up is needed to provide more confirmative information on prognostic value of CD38.

Strengths of this study:

(1) A descriptive study examining SP from China, while existing reports about SP are mainly from the West

(2) Comprehensive assessment of clinical features, survival, and pathological parameters of SP, with semiquantitative immunohistochemical analysis using TMA from the cases

(3) Findings suggesting that CD38 as a potential prognostic marker for SP

Weakness of this study:

(1) Small sample size and short follow-up time

(2) Lack of in depth analysis of NAD+ level in fresh tumor tissues to further elucidate the role of CD38 in modulating NAD + metabolism in the context of solitary plasmacytoma biology

\section{Abbreviations}

CBC, complete blood count; EBER, Epstein-Barr virus-encoded small RNAs; EP, extraosseous plasmacytoma; H\&E, haematoxylin and eosin; MGUS, monoclonal gammopathy of undetermined significance; MM, multiple myeloma; SP, Solitary plasmacytomas; SPB, solitary plasmacytoma of bone.

\section{Author contributions}

Design, review cases, data analysis and writing of manuscript - YL and JG, Design of study, editing and proof of manuscript-SYX, Review cases, data analysis- $\mathrm{CY}$ and JX, Editing and proof of manuscript-PM.

\section{Ethics approval and consent to participate}

This study was approved by the Ethics Committee of Zhongnan Hospital for discarded diagnostic material (no consent required). 


\section{Acknowledgment}

There is no financial conflict of interest to report. We thank Bei Qi for excellent secretarial assistance, and Huan Liu for technical assistance.

\section{Funding}

Department of Science and Technology, Hubei Provincial People's Government (2020CFB343).

\section{Conflict of interest}

The authors declare no conflict of interest.

\section{Availability of data and materials}

Data and materials from this study will be available upon request.

\section{References}

[1] Castillo JJ. Plasma Cell Disorders. Primary Care: Clinics in Office Practice. 2016; 43: 677-691.

[2] Susnerwala S, Shanks J, Banerjee S, Scarffe J, Farrington W, Slevin N. Extramedullary plasmacytoma of the head and neck region: clinicopathological correlation in 25 cases. British Journal of Cancer. 1997; 75: 921-927.

[3] Shih L, Dunn P, Leung W, Chen W, Wang P. Localised plasmacytomas in Taiwan: comparison between extramedullary plasmacytoma and solitary plasmacytoma of bone. British Journal of Cancer. 1995; 71: 128-133.

[4] Pinto JA, Sônego TB, Artico MS, Leal C de FA, Bellotto S. Extramedullary plasmacytoma of the larynx. International Archives of Otorhinolaryngology. 2012; 16: 410-413.

[5] Grammatico S, Scalzulli E, Petrucci MT. Solitary plasmacytoma. Mediterranean Journal of Hematology and Infectious Diseases. 2017; 9: e2017052-e2017052.

[6] Lai C, Hsieh H, Chen H, Chao C, Hua C, Tai C, et al. Clinical Features of Head and Neck Solitary Extramedullary Plasmacytoma in Taiwan. In Vivo. 2020; 34: 261-265.

[7] Basavaiah SH, Lobo FD, Philipose CS, Suresh PK, Sreeram S, Kini $\mathrm{H}$, et al. Clinicopathological spectrum of solitary Plasmacytoma: a single center experience from coastal India. BMC Cancer. 2019; 19: 801.

[8] Lim YH, Park SK, Oh HS, Choi JH, Ahn MJ, Lee YY, et al. A Case of Primary Plasmacytoma of Lymph Nodes. The Korean Journal of Internal Medicine. 2005; 20: 183.

[9] Bartl R, Frisch B, Fateh-Moghadam A, Kettner G, Jaeger K, Sommerfeld W. Histologic classification and staging of multiple myeloma. a retrospective and prospective study of 674 cases. American Journal of Clinical Pathology. 1987; 87: 342-355.

[10] Chang H, Kajal B. Anaplastic variant of plasma cell myeloma with Dutcher bodies. Blood. 2016; 127: 3291.

[11] Kurt H, Ferreira KA. Biphenotypic plasma cell myeloma. Blood. 2019; 133: 1611-1611.

[12] Grogan TM. Plasma cell myeloma marrow diagnosis including morphologic and phenotypic features. Seminars in Diagnostic Pathology. 2003; 20: 211-225.

[13] Stashenko P, Nadler LM, Hardy R, Schlossman SF. Characterization of a human B lymphocyte-specific antigen. Journal of Immunology. 1980; 125: 1678-1685.

[14] Jourdan M, Caraux A, De Vos J, Fiol G, Larroque M, Cognot C, et al. An in vitro model of differentiation of memory B cells into plasmablasts and plasma cells including detailed phenotypic and molecular characterization. Blood. 2009; 114: 5173-5181.

[15] Nadler LM, Ritz J, Hardy R, Pesando JM, Schlossman SF,
Stashenko P. A unique cell surface antigen identifying lymphoid malignancies of B cell origin. The Journal of Clinical Investigation. 1981; 67: 134-140.

[16] Leo R, Boeker M, Peest D, Hein R, Bartl R, Gessner JE, et al. Multiparameter analyses of normal and malignant human plasma cells: CD38++, CD56+, CD54+, $\mathrm{cIg}+$ is the common phenotype of myeloma cells. Annals of Hematology. 1992; 64: $132-139$.

[17] Koda JE, Rapraeger A, Bernfield M. Heparan sulfate proteoglycans from mouse mammary epithelial cells. Cell surface proteoglycan as a receptor for interstitial collagens. The Journal of Biological Chemistry. 1985; 260: 8157-8162.

[18] Freissler E, Meyer auf der Heyde A, David G, Meyer TF, Dehio C. Syndecan-1 and syndecan- 4 can mediate the invasion of OpaHSPG-expressing Neisseria gonorrhoeae into epithelial cells. Cellular Microbiology. 2000; 2: 69-82.

[19] Yao W, Rose JL, Wang W, Seth S, Jiang H, Taguchi A, et al. Syndecan 1 is a critical mediator of macropinocytosis in pancreatic cancer. Nature. 2019; 568: 410-414.

[20] Stewart MD, Ramani VC, Sanderson RD. Shed Syndecan-1 Translocates to the Nucleus of Cells Delivering Growth Factors and Inhibiting Histone Acetylation. Journal of Biological Chemistry. 2015; 290: 941-949.

[21] Akhmetzyanova I, McCarron MJ, Parekh S, Chesi M, Bergsagel PL, Fooksman DR. Dynamic CD138 surface expression regulates switch between myeloma growth and dissemination. Leukemia. 2020; 34: 245-256.

[22] Dattolo P, Allinovi M, Michelassi S, Pizzarelli F. Multiple solitary plasmacytoma with multifocal bone involvement. first clinical case report in a uraemic patient. BMJ Case Reports. 2013; 2013: bcr2013009157.

[23] Warnnissorn N, Treetipsatit J, Limvorapitak W. The cut-offs for kappa/lambda ratio in bone marrow immunohistochemistry for the diagnosis of multiple myeloma. Hematology. 2020; 25: 292 298.

[24] D'Souza L, Bhattacharya D. Plasma cells: you are what you eat. Immunological Reviews. 2019; 288: 161-177.

[25] Lanier LL, Testi R, Bindl J, Phillips JH. Identity of Leu-19 (CD56) leukocyte differentiation antigen and neural cell adhesion molecule. The Journal of Experimental Medicine. 1989; 169: 2233-2238.

[26] Roothans D, Smits E, Lion E, Tel J, Anguille S. CD56 marks human dendritic cell subsets with cytotoxic potential. OncoImmunology. 2013; 2: e23037.

[27] Weber DM. Solitary Bone and Extramedullary Plasmacytoma. Hematology. 2005; 2005: 373-376.

[28] Merza H, Sarkar R. Solitary extraosseous plasmacytoma. Clinical Case Reports. 2016; 4: 851-854.

[29] Kilciksiz S, Karakoyun-Celik O, Agaoglu FY, Haydaroglu A. A review for solitary plasmacytoma of bone and extramedullary plasmacytoma. The Scientific World Journal. 2012; 2012: 895765.

[30] Pham A, Mahindra A. Solitary Plasmacytoma: a Review of Diagnosis and Management. Current Hematologic Malignancy Reports. 2019; 14: 63-69.

[31] Kononen J, Bubendorf L, Kallioniemi A, Bärlund M, Schraml P, Leighton S, et al. Tissue microarrays for high-throughput molecular profiling of tumor specimens. Nature Medicine. 1998; 4: 844-847.

[32] Ye ZY, Xiao SY, Zhao JM, Shi XY, Zheng LR, Wang X, et al. Consensus on tissue detection and pathological diagnosis of enteric Epstein-Barr virus infection. Chinese Journal of Digestion. 2019; 39: 433-437. (In Chinese)

[33] Liu X, He H, Li Y, Huang Y, Li G, Yu Q, et al. The application of antigen receptor gene rearrangement of BIOMED-2 in the pathologic diagnosis of 348 cases with non-Hodgkin lym- 
phoma in a single institution in Southwest of China. Pathology - Research and Practice. 2019; 215: 152615.

[34] Thumallapally N, Meshref A, Mousa M, Terjanian T. Solitary plasmacytoma: population-based analysis of survival trends and effect of various treatment modalities in the USA. BMC Cancer. 2017; 17: 13.

[35] Reinherz EL, Kung PC, Goldstein G, Levey RH, Schlossman SF. Discrete stages of human intrathymic differentiation: analysis of normal thymocytes and leukemic lymphoblasts of T-cell lineage. Proceedings of the National Academy of Sciences of the United States of America. 1980; 77: 1588-1592.

[36] Nakagawara K, Mori M, Takasawa S, Nata K, Takamura T, Berlova A, et al. Assignment of CD38, the gene encoding human leukocyte antigen CD38 (ADP-ribosyl cyclase/cyclic ADPribose hydrolase), to chromosome $4 \mathrm{p} 15$. Cytogenetics and Cell Genetics. 1995; 69: 38-39.

[37] Golden-Mason L, Curry MP, Nolan N, Traynor O, McEntee G, Kelly J, et al. Differential expression of lymphoid and myeloid markers on differentiating hematopoietic stem cells in normal and tumor-bearing adult human liver. Hepatology. 2000; 31: 1251-1256.

[38] Aksoy P, Escande C, White TA, Thompson M, Soares S,
Benech JC, et al. Regulation of SIRT 1 mediated NAD dependent deacetylation: a novel role for the multifunctional enzyme CD38. Biochemical and Biophysical Research Communications. 2006; 349: 353-359.

[39] Aksoy P, White TA, Thompson M, Chini EN. Regulation of intracellular levels of NAD: a novel role for CD38. Biochemical and Biophysical Research Communications. 2006; 345: 13861392.

[40] Gomes AP, Price NL, Ling AJY, Moslehi JJ, Montgomery MK, Rajman L, et al. Declining $\mathrm{NAD}(+)$ induces a pseudohypoxic state disrupting nuclear-mitochondrial communication during aging. Cell. 2013; 155: 1624-1638.

[41] Camacho-Pereira J, Tarragó MG, Chini CCS, Nin V, Escande C, Warner GM, et al. CD38 dictates age-related NAD decline and mitochondrial dysfunction through a SIRT3-dependent mechanism. Cell Metabolism. 2016; 23: 1127-1139.

[42] Yaku K, Okabe K, Nakagawa T. NAD metabolism: Implications in aging and longevity. Ageing Research Reviews. 2018; 47: 117.

[43] Covarrubias AJ, Perrone R, Grozio A, Verdin E. NAD+ metabolism and its roles in cellular processes during ageing. Nature Reviews Molecular Cell Biology. 2021; 22: 119-141 